\title{
A Time Efficient Adaptive Gridding Approach and Improved Calibrations in Five-Hole Probe Measurements
}

\author{
Jason Town and Cengiz Camci \\ Turbomachinery Aero-Heat Transfer Laboratory, Department of Aerospace Engineering, The Pennsylvania State University, \\ University Park, PA 16802, USA \\ Correspondence should be addressed to Cengiz Camci; cxc11@psu.edu
}

Received 25 July 2014; Revised 6 November 2014; Accepted 8 November 2014

Academic Editor: Nekkanti Sitaram

Copyright (C) 2015 J. Town and C. Camci. This is an open access article distributed under the Creative Commons Attribution License, which permits unrestricted use, distribution, and reproduction in any medium, provided the original work is properly cited.

Five-Hole Probes (FHP), being a dependable and accurate aerodynamic tool, are an excellent choice for measuring threedimensional flow fields in turbomachinery. To improve spatial resolution, a subminiature FHP with a diameter of $1.68 \mathrm{~mm}$ is employed. High length to diameter ratio of the tubing and manual pitch and yaw calibration cause increased uncertainty. A new FHP calibrator is designed and built to reduce the uncertainty by precise, computer controlled movements and reduced calibration time. The calibrated FHP is then placed downstream of the nozzle guide vane (NGV) assembly of a low-speed, large-scale, axial flow turbine. The cold flow HP turbine stage contains 29 vanes and 36 blades. A fast and computer controllable traversing system is implemented using an adaptive grid method for the refinement of measurements in regions such as vane wake, secondary flows, and boundary layers. The current approach increases the possible number of measurement points in a two-hour period by $160 \%$. Flow structures behind the NGV measurement plane are identified with high spatial resolution and reduced uncertainty. The automated pitch and yaw calibration and the adaptive grid approach introduced in this study are shown to be a highly effective way of measuring complex flow fields in the research turbine.

\section{Introduction}

Five-Hole Probes are used to determine the three components of the mean velocity vector, local total pressure, and local static pressure [1]. They work by selectively comparing pressure data from five ports on the probe. According to Treaster and Yocum [2], by comparing the pressure differences between these ports, flow velocity magnitude, pitch angle, yaw angle, total pressure, and static pressure can be simultaneously determined. However, this method is found to work in a range of $\pm 30^{\circ}$ of pitch and yaw angle. A method is suggested by Ostowari and Wentz [3] to increase the range to $\pm 85^{\circ}$ by using a nulling method of the probe. However, nulling is not always possible, especially in the tight clearances and rotating machinery such as the flows internal to a turbine research rig. Norwack attempts to increase the range at which a FHP may be used by developing a long, spherical probe [4]. The useable range of the probe is increased to $\pm 65^{\circ}$, but the increased size makes it hard to incorporate in many cases.

Correction methods by interpolation to find the necessary coefficients have been implemented through a variety of methods. A curve fitting approach is used by Treaster and Yocum [2] and Weiz [5]. The curve fitting approach takes into account the fact that the data taken by each port is in a different location. By using an orthogonal grid, the data is curve-fitted across the measurement region and interpolated to the center port of measurement. Reichert and Wendt suggest another method of data reduction for the FHP [6]. This method replaces the pitch and yaw angles with unit vectors and develops a Taylor series based approach to find flow parameters.

Dominy and Hodson studied the effects of Reynolds number extensively. Their study showed how probe design could affect Reynolds number related errors [7]. Treaster and Yocum [2] also covered this feature and suggested that calibration should be made at the expected Reynolds number or a correction factor must be used. Methods for the detection of abnormalities in the probe are suggested by Morrison et al. [8]. These suggestions aid in the identification of probe damage and flow alignment issues.

Investigations into the effect of near-wall measurements with Five-Hole Probes were carried out by Treaster and 
Yocum [2] and Lee and Yoon [9]. They have concluded that measurements should be taken at least two probe diameters away from the wall. Closer distance causes blockage by the probe and acceleration of the flow, leading to greater uncertainty in the measurement. If it is necessary to operate closer than this distance, Lee and Yoon provide guidelines to make such measurements [9].

A FHP might also be used in place of laser Doppler anemometers for wake measurements, Brophy et al. [10]. The main application of the FHP would be in location where it would be difficult to use a laser, such as the rotating frame of reference in a turbine or in geometrically difficult situations to reach flow zones. Sitaram et al. have performed a detailed study on which type of probe to use within the rotating frame of a single stage compressor research rig at the Pennsylvania State University [11]. Town and Camci presented their early observations about using a subminiature Five-Hole Probe in an axial flow turbine rig in [12].

There are many recent studies on the development of FHP based aerodynamic measurement systems. Pisasale and Ahmed [13] presented a theoretical calibration approach for a FHP for highly three-dimensional flows. They also worked on a novel method for extending the calibration range of FHPs for highly three-dimensional flows [14]. Development of a functional relationship between port pressures and flow properties for the calibration and application of a multihole probe to highly three-dimensional flow was a topic of investigation in their 2004 paper [15]. Multihole probes can also be used in the determination of skin friction coefficient in turbulent flows, Lien and Ahmed [16].

The present paper presents significant improvements in FHP based aerodynamic measurements in four significant areas. The specific approach reduces the elapsed calibration time of a typical Five-Hole Probe from 3 hours down to 65 minutes for a $(9 \times 9)$ carpet map of pitch and yaw coefficients because of the unique properties of the new computer controlled calibration mechanism. A second major improvement is in the spatial resolution of measurements in selected high gradient areas such as the boundary layers, wakes, tip vortices, and secondary flow dominated flow zones. The third important property of the present approach is in the improved accuracy of the measurements because of an improved calibration system, the use of more accurate positioning of the probe, the use of highly improved present day transducers, and a careful selection of tubing. Finally, the current approach reduces turbine facility run-time significantly. The new system increases the number of data points that can be collected in a two-hour period from 366 points to 868 points, an increase of $160 \%$.

\section{Symbols}

AFTRF: Axial Flow Turbine Research Facility

$$
C_{P, \text { pitch }} \text { : Pitch coefficient }
$$

$$
\frac{\left(P_{5}-P_{4}\right)}{\left(P_{1}-\bar{P}\right)}
$$

$C_{P, \text { static }}:$ Static pressure coefficient

$$
\frac{\left(\bar{P}-P_{\text {static }}\right)}{\left(P_{1}-\bar{P}\right)}
$$

$C_{P, \text { total }}$ : Total pressure coefficient

$$
\frac{\left(P_{1}-P_{\text {total }}\right)}{\left(P_{1}-\bar{P}\right)}
$$

$C_{P, \text { yaw }}:$ Yaw coefficient

$$
\frac{\left(P_{2}-P_{3}\right)}{\left(P_{1}-\bar{P}\right)}
$$

DAQ: Data acquisition device

$\bar{P}$ : Average value of outside pressure points $(\mathrm{Pa})$

$$
\frac{\left(P_{2}+P_{3}+P_{4}+P_{5}\right)}{(4)}
$$

$P_{1}$ : Pressure point 1 , Figure 1

$P_{2}$ : Pressure point 2, Figure 1

$P_{3}$ : Pressure point 3, Figure 1

$P_{4}$ : Pressure point 4, Figure 1

$P_{5}$ : Pressure point 5, Figure 1

$P_{S}$ : Static pressure at defined location $(\mathrm{Pa})$

$P_{T}$ : Total pressure at defined location $(\mathrm{Pa})$

$P_{\text {static }}:$ Local FHP measured static pressure $(\mathrm{Pa})$

$P_{\text {total }}$ : Local FHP measured total pressure $(\mathrm{Pa})$

$V_{1}$ : Transducer voltage measured at point 1 , Figure 1

$V_{2}$ : Transducer voltage measured at point 2 , Figure 1

$V_{3}$ : Transducer voltage measured at point 3 , Figure 1

$V_{4}$ : Transducer voltage measured at point 4 , Figure 1

$V_{5}$ : Transducer voltage measured at point 5 , Figure 1

$V:$ Absolute velocity magnitude $(\mathrm{m} / \mathrm{s})$

c: Calibration constant $(\mathrm{Pa} / \mathrm{V})$

exit: Measurement location downstream of rotor

inlet: Measurement location upstream of NGV

local: Measurement location in intraspace at specific point

$r$ : Radius to probe traverser track

$u$ : Velocity component in the probe relative $x$-direction $(\mathrm{m} / \mathrm{s})$

$v$ : Velocity component in the probe relative $y$-direction $(\mathrm{m} / \mathrm{s})$

$w$ : Velocity component in the probe relative $z$-direction $(\mathrm{m} / \mathrm{s})$ 


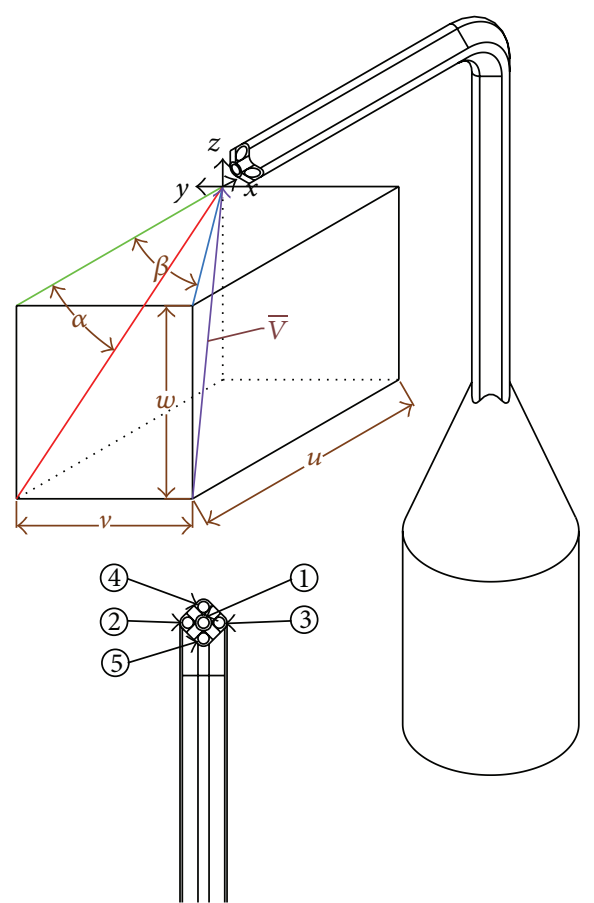

FIgURE 1: Isometric view of a subminiature Five-Hole Probe with velocity vector, positive angles, and positive velocity components and probe port number assignments.

$x$ : Distance of traverser from probe parallel to ground (zero) location

$z$ : Zero voltage measurement $(\mathrm{V})$

$\alpha$ : Probe relative pitch angle $\left({ }^{\circ}\right)$

$\beta$ : Probe relative yaw angle $\left({ }^{\circ}\right)$

$\theta$ : Desired measurement azimuthal angle

$\rho$ : Density $\left(\mathrm{kg} / \mathrm{m}^{3}\right)$.

\section{Material and Methods}

3.1. Objectives. First of the specific objectives is the reduction of elapsed calibration time of a typical FHP for use in the AFTRF from three hours down to about an hour for a $(9 \times$ 9) carpet map of pitch and yaw coefficient. A second major objective is in the improvements in the spatial resolution of measurements in selected high gradient areas such as boundary layers, wakes, tip vortices, and secondary flow dominated zones. The third important objective of the present approach is in the improved accuracy of the measurements. The final objective is about significantly reducing the AFTRF turbine facility run-time for a selected FHP measurement effort.

3.2. Calibration Hardware. A flowchart for the acquisition of calibration data is shown in Figure 2. The commands, interface, and data logging at the computer level are written in LabVIEW. The 16-bit A/D converter system (DAQ) is made by Measurement Computing Corporation (MCC model USB-1608FS). It can obtain $200 \mathrm{k}$ samples per second, interfaces through USB, and has an accuracy of $\pm 0.68 \mathrm{mV}$ at

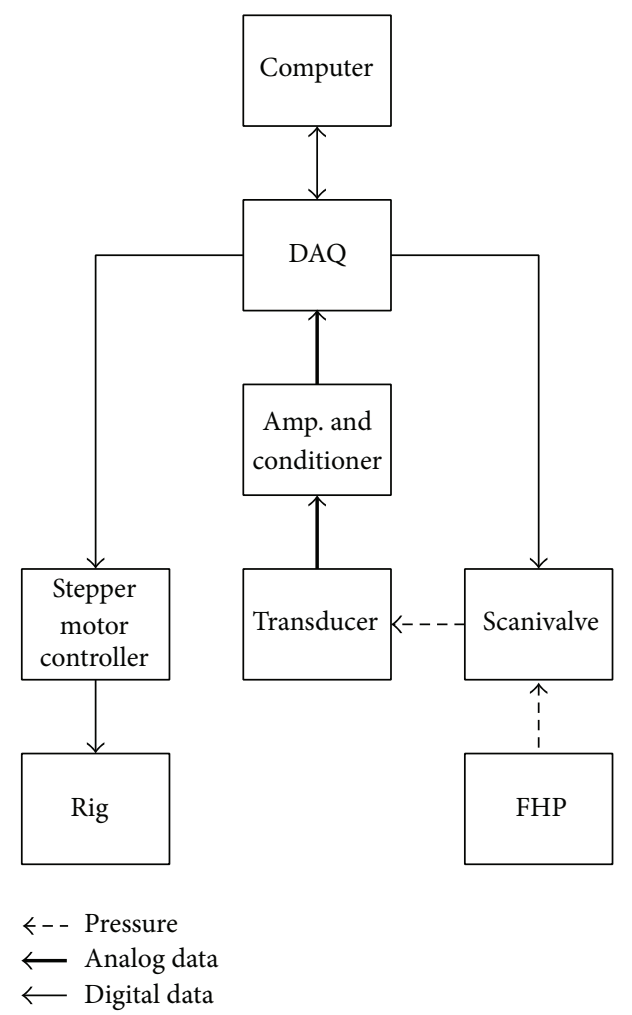

Figure 2: Calibration block diagram for Five-Hole Probe measurements.

an input range of $\pm 1 \mathrm{~V}$. The 16-bit capabilities are essential to help reduce measurement uncertainty during the calibration and measurements.

The pressure is measured by a Validyne DP15 low pressure transducer with a $3500 \mathrm{~Pa}$ diaphragm. The transducer's accuracy is rated at $0.25 \%,( \pm 3.5 \mathrm{Pascal})$ of the full-scale measurement. Only one transducer is used to measure all five ports; its reference port is left open to atmosphere, and efforts are made to isolate the transducer thermally, electronically, and mechanically. Using one transducer for all five ports eliminates the bias errors coming from individual transducer zero values.

The transducer is connected to a Scanivalve Corp. 48channel mechanical pressure selector. The specific electrical commands to step and reset the scanner are provided by the digital output D/A of the DAQ. The mechanical scanning approach with one transducer reduces measurement uncertainty by canceling out any thermal shift and calibration error the transducer might measure. It also reduces the total cost of the system but comes up with increased measurement time for the calibrations and measurements.

Previously employed methods of pitch and yaw calibration were applied by hand. To increase the accuracy of movements and decrease the total time taken for a complete calibration map, computer actuated rotary tables for pitch and yaw movements are used. Two different rotary tables are used, both provided by Velmex Inc. The larger of the two, model B4800TS, is used to change the pitch angle. For each 


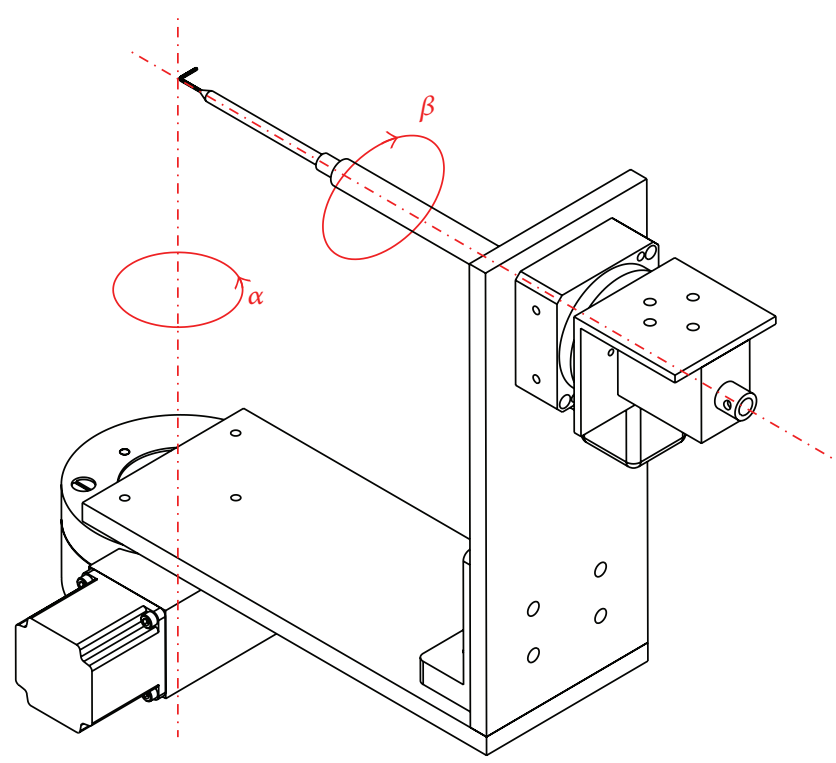

FIgURE 3: Calibrator design with pitch $(\alpha)$ and yaw $(\beta)$ movements.

half step it moves $0.0125^{\circ}$. A second table, model B5990TS, is used to for yaw adjustments. For each half step it moves $0.010^{\circ}$.

The calibration rig shown in Figure 3 is designed to minimize movement of the probe tip in the direction normal to the outlet of the wind tunnel. The design intent is to keep the probe tip as near the intersection of the pitch and yaw axes as possible. This reduces the arm length of the probe and its displacement within the calibration jet.

A subminiature FHP shown in Figure 1 is designed and created on site using five hypodermic needles. It has a square cross-section with one hole in the center surrounded by four others located above, below, left, and right of the center. All sides of the probe have been beveled to a $45^{\circ}$ angle, leaving the center port in a normal plane to the flow. The specific hypodermic needles reduce the size of the probe to a maximum diameter of $1.68 \mathrm{~mm}$ at the tip. The inherently small diameter of hypodermic tubing and the long length of tubing lead to a very large length to diameter ratio. Subminiature FHPs usually require relatively long settling and data acquisition times. However, the small tip size increases the spatial resolution of the measurements and allows the probe to be inserted into the complex internal flow areas of most turbomachinery passages. An additional Pitot probe is also used to measure the calibration tunnel's free jet axial velocity, total pressure, and static pressure.

The calibration facility, shown in Figure 4, consists of an open loop wind tunnel with an axial blower, a diffused housing with multiple screens, a plenum chamber, a high area ratio circular nozzle, a circular to square transition nozzle, and a section of constant cross-sectional duct. The compressor is $45.7 \mathrm{~cm}$ in diameter and is driven by a variable speed motor rated up to $7.5 \mathrm{~kW}$. The tests are performed in the free jet just outside the constant cross-sectional duct. Free jet velocities are continuously adjustable via an AC inverter up to
$28 \mathrm{~m} / \mathrm{s}$. Turbulent flow characteristics in the test section can be adjusted to turbulence intensity values between $0.5 \%$ and $1.2 \%$ by the use of calibrated screens and biplane turbulence promoters. Details of the test section flow quality can be found in Wiedner [1], Kuisoon et al. [17, 18], and Camci and Rizzo [19].

3.3. Calibration Technique. A modified version of the calibration/reduction technique of a nonnulling FHP used by Treaster and Yocum [2] is used in this paper. The main difference between the two methods is in the way pitch and yaw angles are defined. Figure 1 defines the positions of the holes, the coordinate system for the probe, the positive angles of the probe, and the positive velocity components. In this configuration, being positioned on the probe facing the incoming flow, a positive pitch value would occur when the flow was coming from below the probe (nose up). Positive yaw value would occur when the flow is coming from the left (nose right). The only manual input into the calibration sequence is the initial hand positioning of the probe at zero pitch, zero yaw angles. The calibration grid uses an improved 81-point $(9 \times 9)$ configuration with more data points in the nonlinear region near the maximum acceptance range of the probe $\left( \pm 30^{\circ}\right)$. Calibration maps for coefficients of yaw, pitch, total pressure, and static pressure are calculated directly from the measurements of the FHP and Pitot probe as shown in Figures 8,9 , and 10. In most earlier studies, the carpet maps were limited to a 49 -point $(7 \times 7)$ configuration or less. One of the main concerns with calibrating a FHP is producing a high quality map with reduced absolute error. One concern is about instant changes in calibration flow quality that can be caused by laboratory disturbances or unwanted air currents. Another source of error can occur when the probe is first aligned with the flow. The probe is initially aligned by hand and is prone to human error. Only one transducer is used to sample pressure from all pressure measurement points. Five input channels to the rotary pressure scanner are required for the FHP ports and two input channels are needed for the Pitot probe documenting the total pressure and static pressure in the test section.

Using a single transducer for all seven pressure measurements during calibration measurably increased the elapsed time for a calibration. However, this approach significantly reduces calibration error. Equation (6) states that pressure is a function of the measured voltage $V_{n}$, the zero $z$, and a calibration factor $c$. Since the zero and the calibration factor are for only one transducer, they can be considered constant for all pressure measurements. The analysis will only take $C_{P \text {,total }}$ from (3) into consideration, though it can be done with any of the other pressure coefficient equations. Substitution of (6) into (3) results in (7). Since $c$ is in every term, canceling it out leads to (8). The result of canceling out the $z$ term in the numerator and simplifying the $z$ term in the denominator is shown in (9). Finally, the $z$ term in the denominator is cancelled out and the result is shown in (10). The result shows that when calculating $C_{P \text {,total }}$, or for that matter any of the $C_{P}$ values, the zero and calibration factors cancel out. Thus, using one transducer eliminates the source of error that could be 


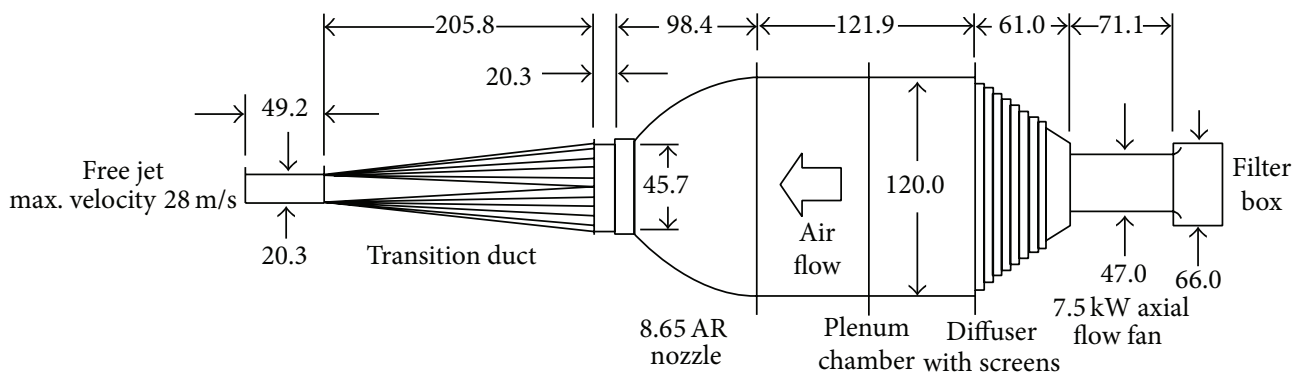

FIGURE 4: Calibration wind tunnel, dimensions in centimeters (not to scale).

caused by a typical calibration of a transducer and incorrect zeroing procedure. Consider

$$
P_{n}=\left(V_{n}-z\right) c,
$$

$$
\begin{aligned}
& C_{P, \text { total }} \\
& =\left(\left(V_{1}-z\right) c-\left(V_{T}-z\right) c\right) \\
& \times\left(\left(V_{1}-z\right) c\right. \\
& \left.\quad \quad \quad \frac{\left(V_{2}-z\right) c+\left(V_{3}-z\right) c+\left(V_{4}-z\right) c+\left(V_{5}-z\right) c}{4}\right)^{-1}, \\
& C_{P, \text { total }} \\
& =\left(\left(V_{1}-z\right)-\left(V_{T}-z\right)\right) \\
& \quad \times\left(\left(V_{1}-z\right)\right. \\
& \left.\quad-\frac{\left(V_{2}-z\right)+\left(V_{3}-z\right)+\left(V_{4}-z\right)+\left(V_{5}-z\right)}{4}\right)^{-1}, \\
& \quad C_{P, \text { total }}=\frac{V_{1}-V_{T}}{\left(V_{1}-z\right)-\left(V_{2}+V_{3}+V_{4}+V_{5}-4 z\right) / 4}, \\
& \quad C_{P, \text { total }}=\frac{V_{1}-V_{T}}{V_{1}-\left(V_{2}+V_{3}+V_{4}+V_{5}\right) / 4} .
\end{aligned}
$$

3.4. Calculating Unknown Flow Variables. A FORTRAN code developed in-house is the traditional method of data reduction in the measurements of a FHP. The recent fully automated LabVIEW implementation of the same analytical calibration/reduction procedure is shown to produce identical results or better when compared to our past manual calibration/reduction system. Input to the code requires the current ambient temperature $(T)$, absolute static pressure $\left(P_{S}\right)$, and the five pressures as measurements of the FHP. The program then determines the pitch angle $\alpha$ through a series of linear interpolations. The interpolation scheme calls $C_{P \text {,pitch }}$ to be calculated for each possible pitch angle in the 81point carpet map where there are nine possible pitch angles at a constant $C_{P, \text { yaw }}$. A second interpolation calculates the value of pitch angle by using the measured value of $C_{P, \text { pitch }}$. Yaw angle $\beta$ is calculated in a similar interpolation scheme. This interpolation scheme calls $C_{P \text {,yaw }}$ to be calculated for each possible yaw angle at a constant $C_{P \text {,pitch }}$. The next interpolation calculates the exact value of yaw angle by using the measured value of $C_{P \text {,yaw }}$. A calibration chart used to visualize the data of the interpolations of pitch and yaw angle can be found in Figure 8 .

Pitch and yaw angles are used for the interpolation of $C_{P \text {,total }}$ and $C_{P, \text { static }}$. The interpolation works similarly to pitch and yaw interpolation. First, $\alpha$ is held constant and an array of $C_{P \text {,total }}$ or $C_{P \text {,static }}$ is found with an array of $\beta$. The previously found value of $\beta$ is then used to calculate $C_{P \text {,total }}$ and $C_{P, \text { static }}$, respectively. The charts used for the calculation of $C_{P \text {,total }}$ and $C_{P \text {,static }}$ can be found in Figures 9 and 10. Hence,

$$
\begin{gathered}
P_{\text {total }}=P_{1}-C_{P, \text { total }}\left(P_{1}-\bar{P}\right), \\
P_{\text {static }}=\bar{P}-C_{P, \text { static }}\left(P_{1}-\bar{P}\right), \\
V=\left[\frac{2\left(P_{T}-P_{S}\right)}{\rho}\right]^{1 / 2}, \\
u=V \cdot \cos \alpha \cdot \cos \beta, \\
v=V \cdot \sin \beta, \\
w=V \cdot \sin \alpha \cdot \cos \beta .
\end{gathered}
$$

Equation (3) can be rewritten to solve for total and static pressure. The results are shown in (11) and (12). Pitch and yaw angles are the first values found in the data reduction and are known. Definitions of velocity components with conventions are given in Figure 1 and derivations are in (14), (15), and (16).

3.5. Axial Flow Turbine Research Facility. The Axial Flow Turbine Research Facility at Pennsylvania State University currently consists of a single stage state-of-the-art HP turbine with 29 nozzle guide vanes (NGV) and 36 rotor blades. Figure 5 shows a cross-sectional view of the AFTRF. Flow enters through the inlet bellmouth and accelerates through the NGV. A detailed explanation of the design and characteristics of the AFTRF can be found in Lakshminarayana et al. [20].

The recent probe traverser as shown in Figures 5 and 6 is a modification of a previous design used in the AFTRF. In the previous design, circumferential movements were achieved by a belt system. The flexible belt system had noticeable play in it, and the new system replaces the belts 


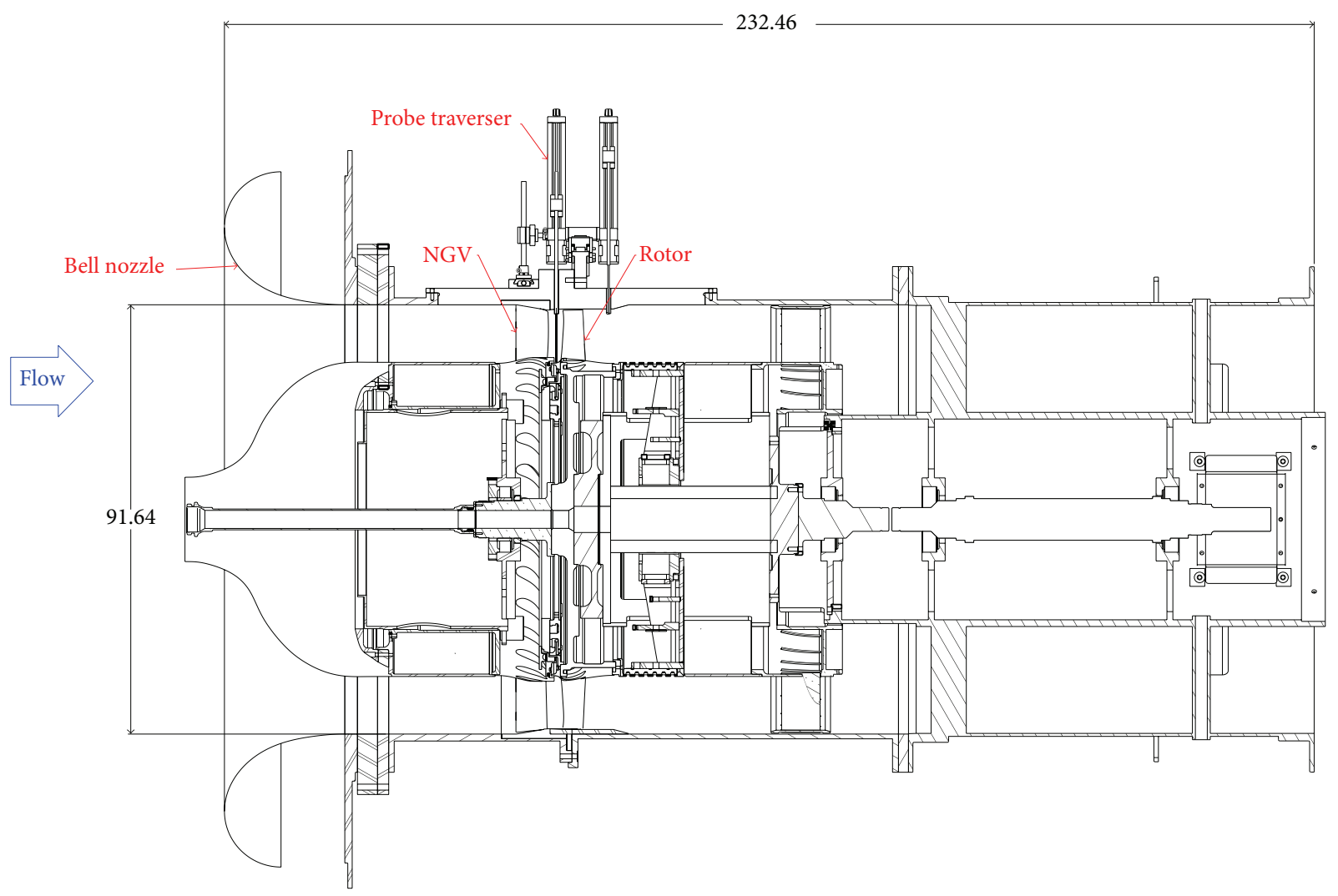

FIgURe 5: Axial Flow Turbine Research Facility (AFTRF) cutaway (dimensions in cm).

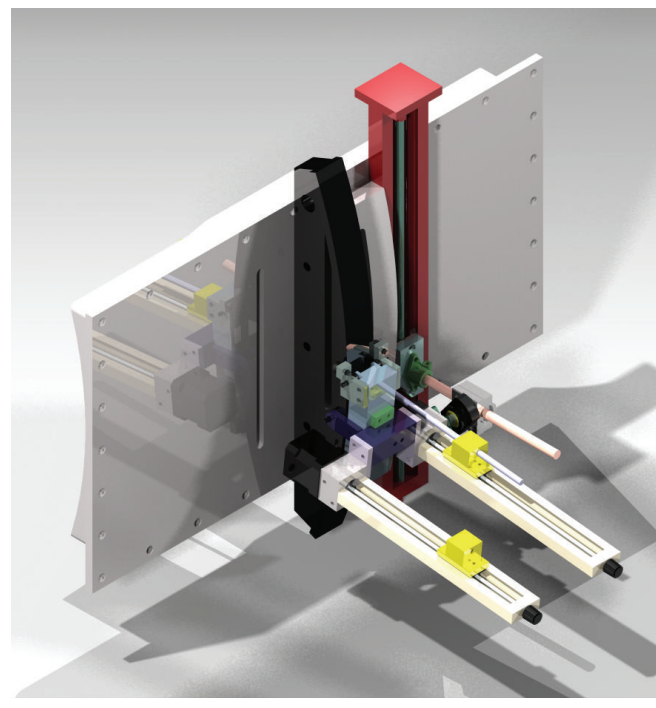

FIGURE 6: Probe traverser attached to the AFTRF instrumentation window, isometric view.

with a precision built linear traverser shown in red. Replacing the belt system with a traverser greatly increases the accuracy and reduces the play of the system. There are two radial traversers that are connected to the trolley (light blue), and the probe holders are shown in yellow. It is designed with two radial traversers so that the system could take measurements in locations in between the NGV and rotor (intraspace), or downstream of the rotor. The new traversing system also includes improvements to the stepper motor drivers, which helps the traverser to move much faster than previously.

A cylindrical coordinate system is used within the AFTRF. Movements of the trolley could be translated to azimuthal position by calculation of arc length. Equation (17) is the result of the calculation to find the distance the traverser must move to change to a particular azimuthal angle $\theta$. All distances are derived from a zero angle; the probe is parallel to the normal of the traverser. The radius $r$ is the distance to the surface of the track that the trolley rides upon. The final variable, $x$, is the distance that the traverser must move from the zero position. To move from $1^{\circ}$ to $2^{\circ}, x$ is calculated for both cases and the values are subtracted to find the correct distance:

$$
x=\frac{r \cdot \tan \theta}{\sqrt{\tan ^{2} \theta+1}} .
$$

Data is acquired by a modular National Instruments CompactDAQ system. The module used to measure the analog signals of the Validyne DP-15 pressure transducers is a 32channel, 16-bit NI 9205 data acquisition system. Rotor speed measurements are obtained from a high resolution MIL-spec optical encoder from BAE Systems (Model H25) encoder attached to the rotor. A Velmex VXM drives the stepper motors. All data logging, traverser movements, data analysis, 


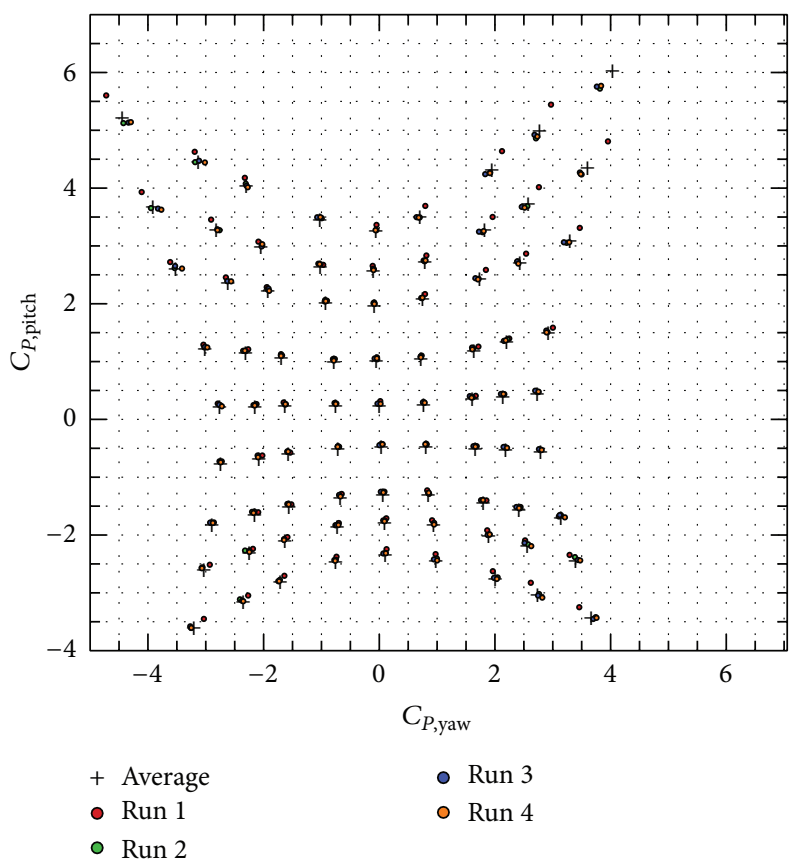

Figure 7: Average values (crosses) with data spread of four runs (points).

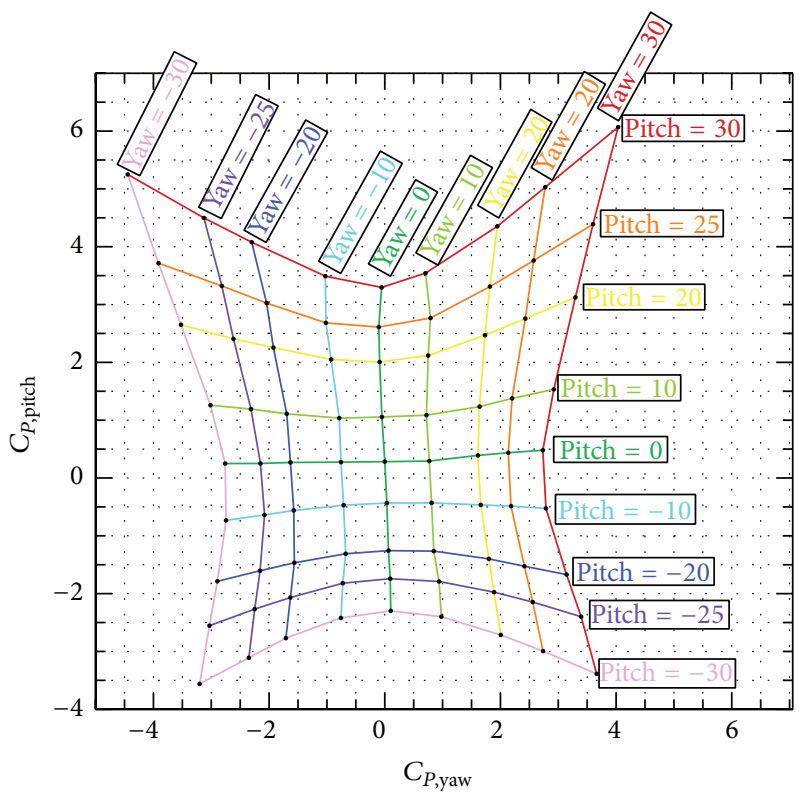

FIGURE 8: Coefficient of yaw and pitch with lines of constant pitch or yaw angles, $81(9 \times 9)$ points.

and data recording are completed by a custom LabVIEW script.

Although a conventional Scanivalve mechanical pressure scanner was used in the calibration of the FHP measurements for the improved calibration accuracy, our current AFTRF FHP measurements are performed using state-of-the-art electronic pressure scanners. Thirty-two-channel ZOC22b units from Scanivalve Corporation were employed in most

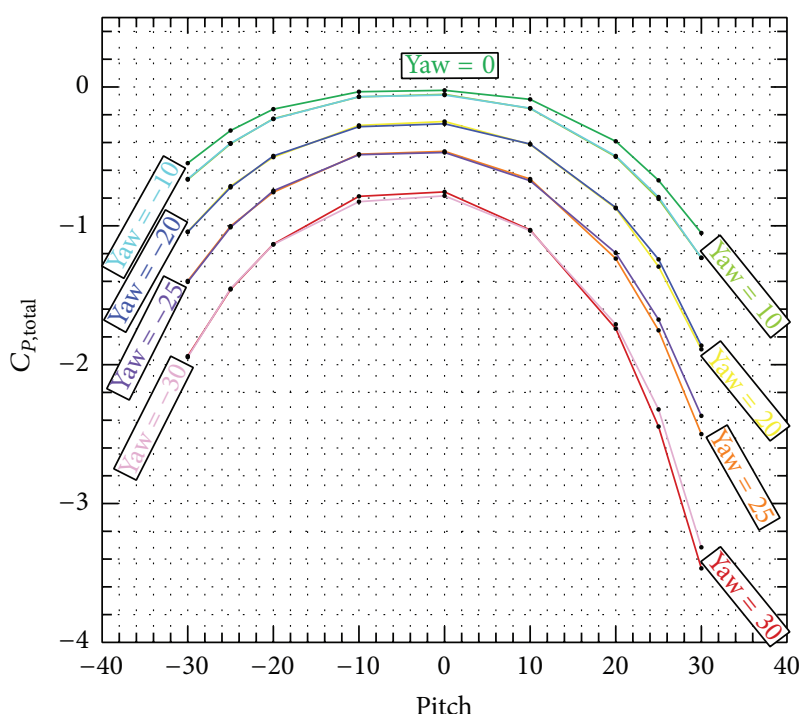

FIGURE 9: Pitch and coefficient of total pressure with lines of constant yaw, $81(9 \times 9)$ points.

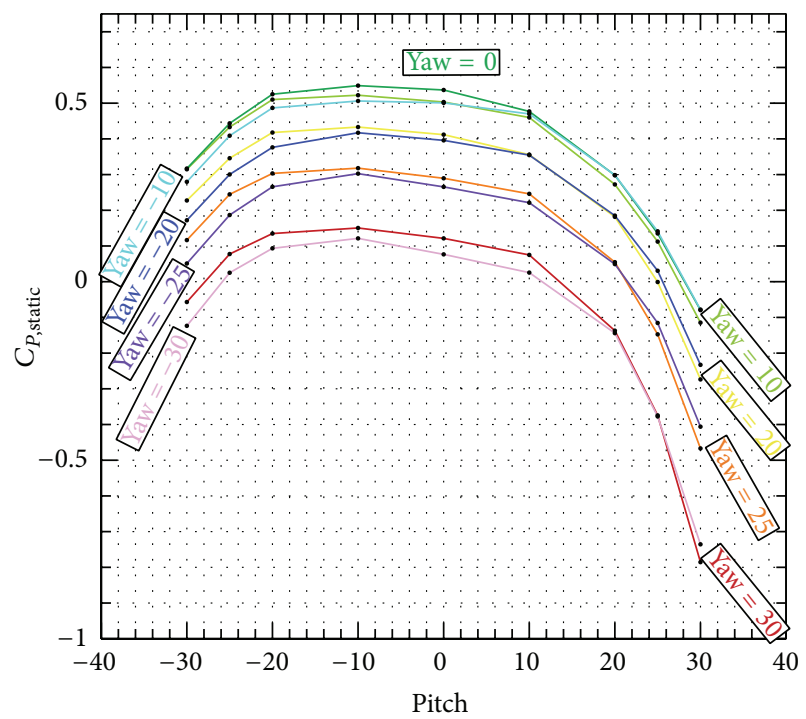

Figure 10: Pitch and coefficient of static pressure with lines of constant yaw, $81(9 \times 9)$ points.

of the turbine runs for faster operation and better thermal stability of the measurements.

Calculation of density in our FHP based calibrations and actual measurements require a measurement of local temperature. A K type thermocouple based probe is employed in our temperature measurements throughout the AFTRF. The thermocouple signal is referenced/amplified and converted into engineering units in a custom NI-9213 thermocouple processing unit. Our final temperature measurement accuracy for calibrations and AFTRF is estimated to be around $\pm 0.15^{\circ} \mathrm{C}$.

Fluid flow through the AFTRF varies in velocity to the extent that a probe calibrated for the inlet could not be used 
TABle 1: Predicted midspan flow speeds and locations.

\begin{tabular}{|c|c|c|c|c|}
\hline Location & Inlet & Intraspace & Absolute exit & Relative exit \\
\hline $\begin{array}{l}\text { Midspan velocity } \\
(\mathrm{m} / \mathrm{s})\end{array}$ & 16.15 & 66.30 & 20.05 & 66.88 \\
\hline
\end{tabular}

in the intraspace or rotor exit relative frame locations but could be used in the rotor exit absolute frame of reference. Table 1 covers the values of midspan velocity in meters per second. Present measurements are performed in the intraspace location, so the probe is calibrated at about $66 \mathrm{~m} / \mathrm{s}$.

\section{Results}

4.1. Five-Hole Probe Calibration. The carpet map as shown in Figure 7 was obtained four times in subsequent runs in an effort to establish the repeatability of the calibration process. The center of each cross represents the average value, while the four points surrounding each cross represent the data collected from each run. Nearly all points within the $\pm 20^{\circ}$ range have good grouping and are close to the average value. In the outlying regions of the calibration, those greater than $\pm 20^{\circ}$, the grouping is not as tight and initial alignment errors are exacerbated. The star shaped carpet map shown in Figure 7 is not perfectly symmetrical because a dimensionally perfect and symmetrical FHP is very difficult to manufacture because of the probe's small size and the inherent machining imperfections.

Calculations of $C_{P \text {,pitch }}$ and $C_{P \text {,yaw }}$ are found directly through (4) and (1). The results are used to interpolate pitch and yaw angle values with the help of the data presented in Figure 8 that is a typical averaged carpet map produced by the current automated calibration approach. Figure 9 presents the variation in $C_{P \text {,total }}$ with respect to pitch and yaw angle. Interpolation is done to find $C_{P \text {,total }}$ using the pitch and yaw found in the previous section. Similarly, Figure 10 provides $C_{P \text {,static }}$ as a function of pitch and yaw. $C_{P \text {,static }}$ can be recovered from the FHP measurements using the previously found pitch and yaw angles.

Previous design of calibration called for the probe to be moved by hand. One of the major advantages of the current computer automated system is stepper motor driven movements. No longer relying on human movement allows for a much greater degree of accuracy. The stepper motor controller can move the turntables in steps as fine as $0.0125^{\circ}$. Once the initial zero pitch and zero yaw position are defined at the start of each run, the computer driven mechanism can move to a new position with excellent spatial resolution and accuracy.

Calibrations of a FHP by manual pitch and yaw angle adjustments are long and arduous tasks. Previously, a 49point map in a manual calibration effort took at least three hours to complete since a high quality adjustment of each pitch and yaw angle required great care. When the design was changed to the current automated system, a 49-point map took 65 minutes, and an 81-point map took 100 minutes. Further reduction in elapsed time is seen if multiple transducers are used, at the cost of previously mentioned accuracy improvements with the single transducer approach. An 81point grid with multiple transducers takes approximately 22 minutes to complete. Ensemble averaging from four individually obtained carpet maps can also be very easily obtained in the current computer driven system in a time efficient manner. Ensemble averaging is an excellent way of removing some of the error originating from initial alignment of the probe. Paying attention to properly recording the transducer zero voltages just before a turbine run starts is an effective way of improving accuracy in the multiple transducer approach.

The method of attachment of a FHP to the pitch and yaw calibrator is extremely critical. The style and quality of the mechanical attachment influence the movement of the probe and the value of the calibration. Large swings and displacements can produce large errors in the calibration from spatial nonuniformity, turbulence decay, or shear layer mixing. The calibrator in Figure 3 shows a design where the probe's tip is located near the intersection of the pitch and yaw rotational axes. The improved design makes the calibration more accurate and reduces the uncertainties.

Due to the nature of the design of the probe, the initial alignment must be performed by hand. This is because of the small size and unforeseen defects that make nulling the probe unfeasible. However, a few techniques are developed in order to increase the initial alignment accuracy. First, a plumb bob is used to align the calibrator base so that it is parallel to the exit of the wind tunnel. This helps to insure that the probe will be held normal to the exit of the wind tunnel and parallel to the streamlines in the pitch direction. Yaw angle alignment is done with the help of a visible, horizontal laser beam. Placing a piece of paper over the exit of the tunnel allows for comparison of the shape of the shadow of the probe. The shadow is then brought to its minimum size by making small adjustments with the yaw stepper motor. These two alignments are done at the beginning of every run.

An uncertainty analysis is prepared for the total pressure, the static pressure, and velocity as defined by (11), (12), and (13). An adaptation of a method set forth by Taylor [21] is used. Equation (18) is an example of the total pressure uncertainty estimates. The other variables follow the same processes but are not presented for brevity:

$$
\begin{gathered}
\delta P_{T} \\
=\left[\left(\delta P_{1} \frac{\delta P_{T}}{\delta P_{1}}\right)^{2}+\left(\delta P_{2} \frac{\delta P_{T}}{\delta P_{2}}\right)^{2}+\left(\delta P_{3} \frac{\delta P_{T}}{\delta P_{3}}\right)^{2}\right. \\
+\left(\delta P_{4} \frac{\delta P_{T}}{\delta P_{4}}\right)^{2}+\left(\delta P_{5} \frac{\delta P_{T}}{\delta P_{5}}\right)^{2} \\
\left.+\left(\delta C_{P, \text { total }} \frac{\delta P_{T}}{\delta C_{P, \text { total }}}\right)^{2}\right]^{1 / 2}, \\
\delta P_{1}=\delta P_{2}=\delta P_{3}=\delta P_{4}=\delta P_{5}= \pm 5 \mathrm{~Pa} \\
\delta C_{P, \text { total }}= \pm \frac{1}{2}\left(C_{P, \text { total max }}-C_{P, \text { total min }}\right) .
\end{gathered}
$$




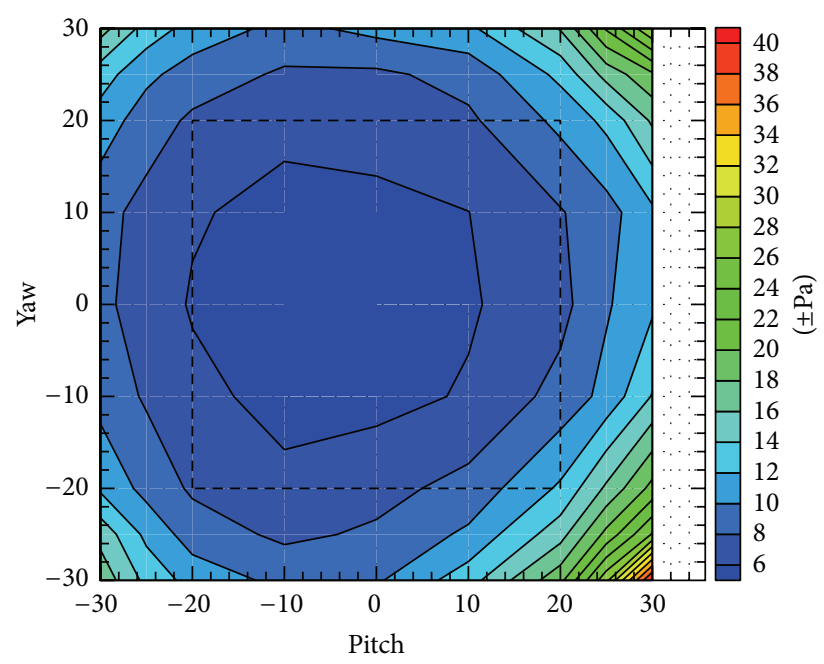

FIGURE 11: Total pressure uncertainty at $20 \mathrm{~m} / \mathrm{s}, 81(9 \times 9)$ points (in terms of $\pm \mathrm{Pa}$ ).

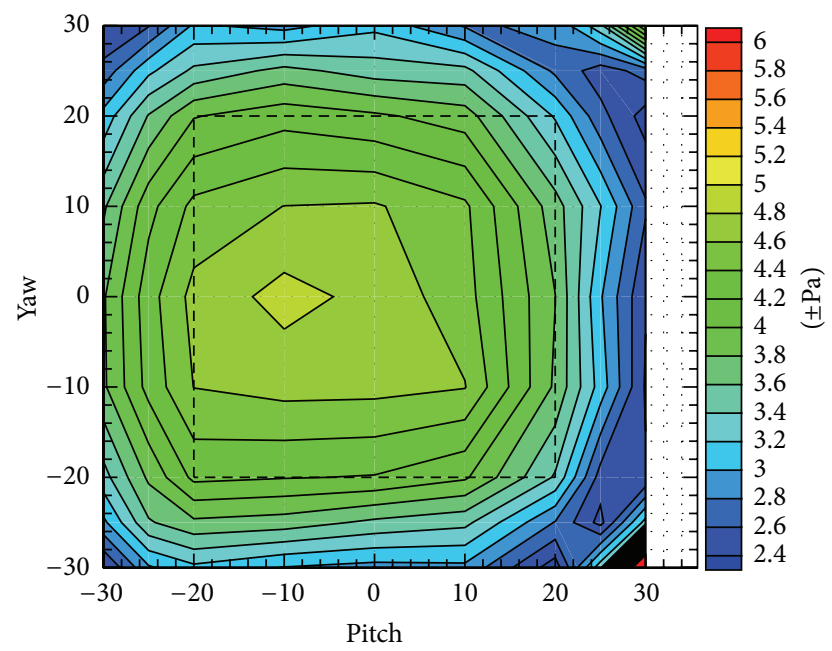

FIGURE 12: Static pressure uncertainty at $20 \mathrm{~m} / \mathrm{s}, 81(9 \times 9)$ points (in terms of $\pm \mathrm{Pa}$ ).

Accuracy of the transducer is approximately $\pm 3.5 \mathrm{~Pa}$. Due to additional equipment and the high length to diameter ratio of connecting tubes, the accuracy decreased to $\pm 5 \mathrm{~Pa}$. This is expressed in (19). The uncertainty of the total pressure coefficient needs to be estimated using (20).

Total pressure uncertainty is shown in Figure 11 where the most accurate region of the measurement is located slightly to the left of the zero pitch, zero yaw location. This is due to small defects that are asymmetries in the probe tip shape. The $30^{\circ}$ pitch, $-30^{\circ}$ yaw location sees a much larger variation in total pressure. The dashed box represents a $\pm 20^{\circ}$ region that is to help identify the range for which the probe is most accurate. When taking measurements, the incidence angle of the flow to the probe is kept within this region.

Static pressure uncertainty at $20 \mathrm{~m} / \mathrm{s}$ is shown in Figure 12. The uncertainty results are not symmetric due to imperfections in the probe. The uncertainty near the center

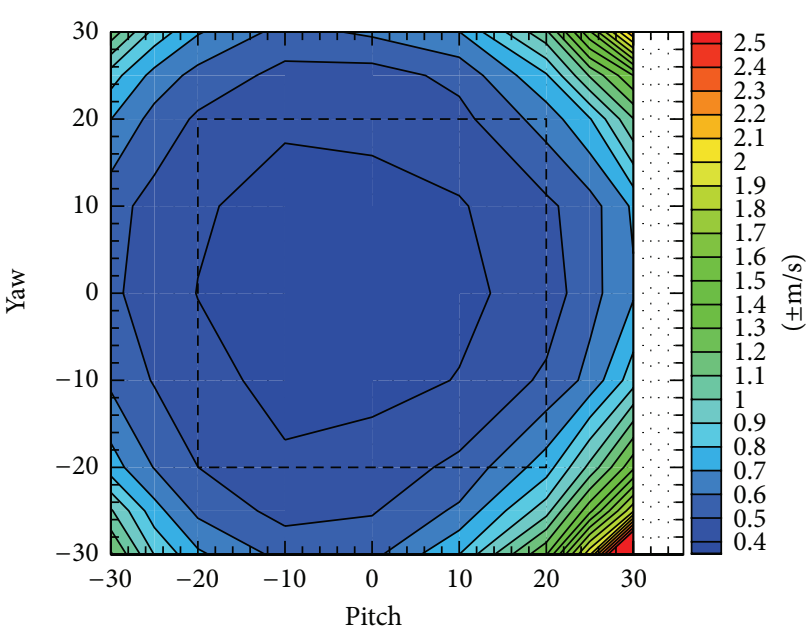

FIGURE 13: Velocity uncertainty at $20 \mathrm{~m} / \mathrm{s}, 81(9 \times 9)$ points (in terms of $\pm \mathrm{m} / \mathrm{s})$.

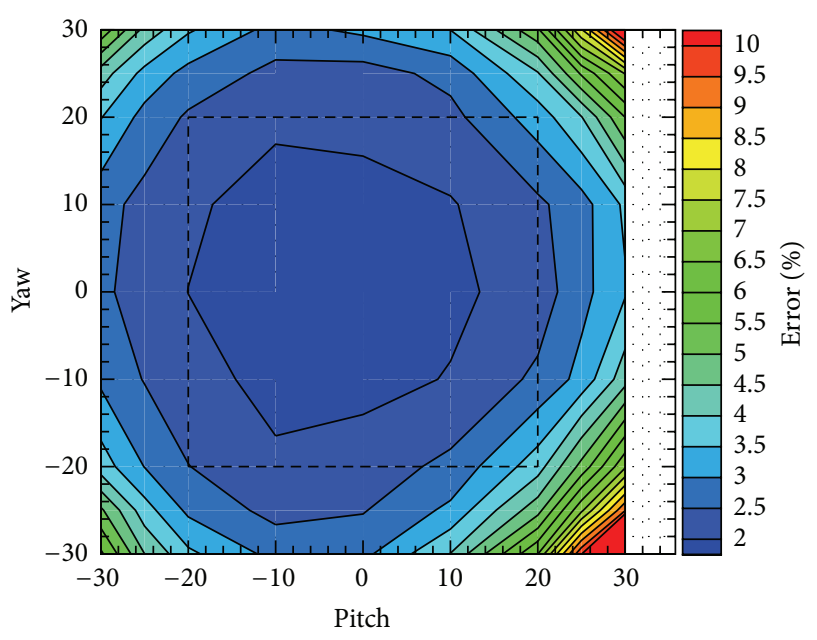

FIGURE 14: Velocity percent error at $20 \mathrm{~m} / \mathrm{s}, 81(9 \times 9)$ points (in terms of percentage from baseline).

is larger. This is most likely because of the way the static pressure is calculated. The probe measures greater pressure when it is aligned to flow leading to an increase in uncertainty through greater measurement variability. When the probe is taking measurements near the maximum range, some of the pressure measurements decrease and the overall uncertainty is reduced.

Velocity uncertainty shown in Figure 13 has a minimum to the left of center. It reaches a maximum uncertainty greater than $\pm 2.5 \mathrm{~m} / \mathrm{s}$ at pitch $30^{\circ}$ and yaw $-30^{\circ}$. Uncertainty in the recommended $\pm 20^{\circ}$ range is below $\pm 0.8 \mathrm{~m} / \mathrm{s}$.

Figure 14 represents the relative velocity uncertainty. The smallest error is located to the left of center in the map, while the largest error is located near the edges. At pitch $30^{\circ}$, yaw $-30^{\circ}$, the error is greater than $10 \%$. Within the dashed box range of $\pm 20^{\circ}$ no error is found to be greater than $4 \%$. The estimated error in the range of $\pm 10^{\circ}$ is about $2 \%$ of the calibration tunnel velocity. 


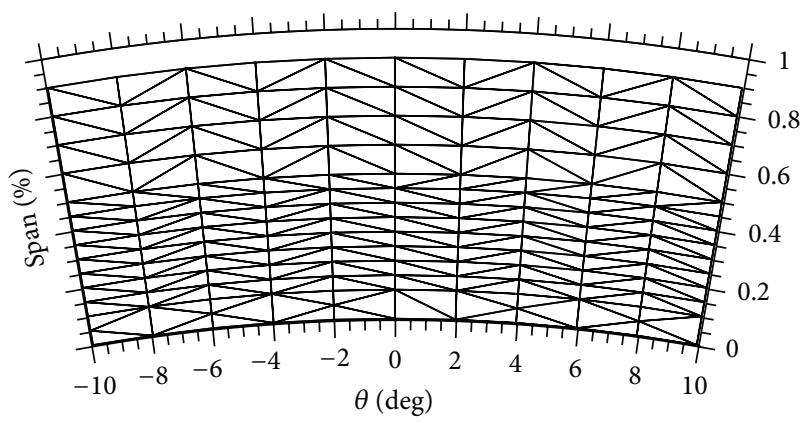

FIGURE 15: Initial measurement grid, 165 points.

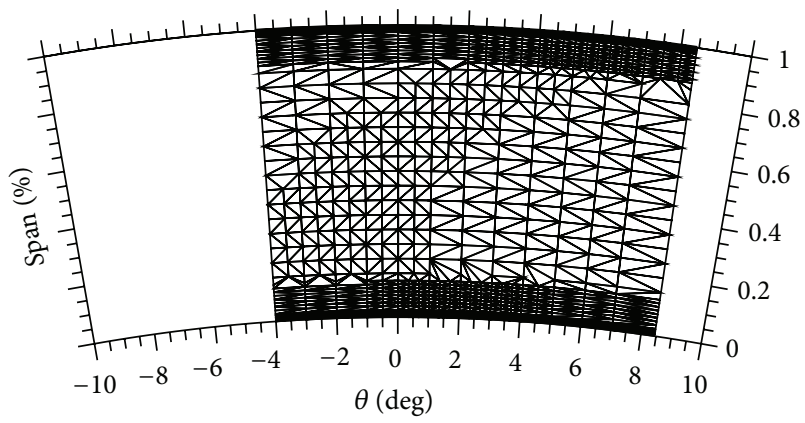

FIGURE 16: Refined measurement grid, 868 points.

The preceding figures determine that the most ideal operation of this probe would be within the $\pm 20^{\circ}$ range that is defined by the dashed box. A pitch and yaw range of $\pm 30^{\circ}$ is also possible. However, the elevated measurement uncertainty should be carefully evaluated in this range.

4.2. AFTRF Intraspace Five-Hole Probe Measurement Results. The new traverser greatly reduced the amount of time it takes to move from one point to another. The maximum allowable testing period for the AFTRF was about two hours. In older previous experiments performed in the AFTRF, a 336point mesh was used with the same sampling time for each measurement point. This mesh would take approximately two hours to complete and could not handle adaptive gridding. Improvements in the traversing system increased the number of points that are measured in a two-hour period to 868 . The adaptive mesh and traverser improvements increased the number of points taken by nearly $160 \%$ over the previously used meshing techniques for the same two-hour test.

The addition of the adaptive grid approach into the mesh generation allows fine measurements in locations of greater interest and large gradients. The iterative process that is done for the adaptive gridding is currently manual. The initial grid of 165 points is shown in Figure 15. This grid is refined in areas of wake, boundary layer, and endwall vortices until the grid becomes what is shown in Figure 16. The refined grid of Figure 16 allows for less time to be spent in regions of

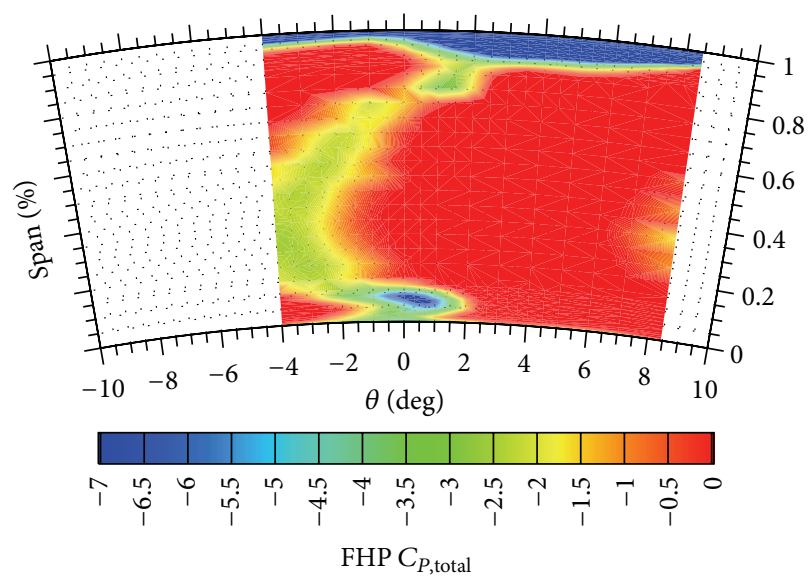

FIGURE 17: FHP coefficient of total pressure, intraspace.

less variation and more time spent in large gradient areas. Consider

$$
\begin{aligned}
C_{P, \text { total }} & =\frac{P_{T, \text { local }}-P_{T \text { inlet }}}{(1 / 2) \rho V_{\text {inlet }}^{2}}, \\
C_{P, \text { static }} & =\frac{P_{S, \text { local }}-P_{T, \text { inlet }}}{(1 / 2) \rho V_{\text {inlet }}^{2}} .
\end{aligned}
$$

Coefficient of total pressure as defined by (21) for one vane pitch $\left(12.41^{\circ}\right)$ is shown in Figure 17. In this figure, the more negative values "going toward blue" indicate greater total pressure loss. The boundary layer near the hub is very small. The probe at the zero span location is currently behind a backward facing step which reduces size of the boundary layer. The passage vortex of the hub centered in the blue region is in the range of $\theta$, from $-1^{\circ}$ to $1^{\circ}$, and span 0.05 to 0.10 . The vane wake is the yellow region of the total pressure loss that curves through the measurement plane. Above $90 \%$ span, the uncertainty is increased as the probe is nearing the slot. This slot is used to access the intraspace measurement plane and the boundary layer can be seen along with the casing passage vortex.

Coefficient of static pressure measured by the FHP in the intraspace of the AFTRF as defined by (22) is shown in Figure 18. One can see the effects the secondary flows in Figures 17 and 18. The highest static pressure can be found near the hub; the lowest static pressure is found near the casing. The casing has greater uncertainty because the measurements are taken near the instrumentation slot. Static pressure over the midspan varies from being values of -23 to values of -28 .

Velocity magnitude measured by the FHP is shown in Figure 19. The highest velocity is found near the hub and velocity is reduced near the casing. The blue region in Figure 17 identified as the hub passage vortex appears here as a yellow deficit zone in velocity in the same location. Inspection can make out the outline of the vane wake, but it is not as clear as the hub passage vortex. Velocities are the lowest near the casing. This is an area of increased uncertainty due to its proximity to the instrumentation slot. The casing passage vortex and boundary layer can be identified. 

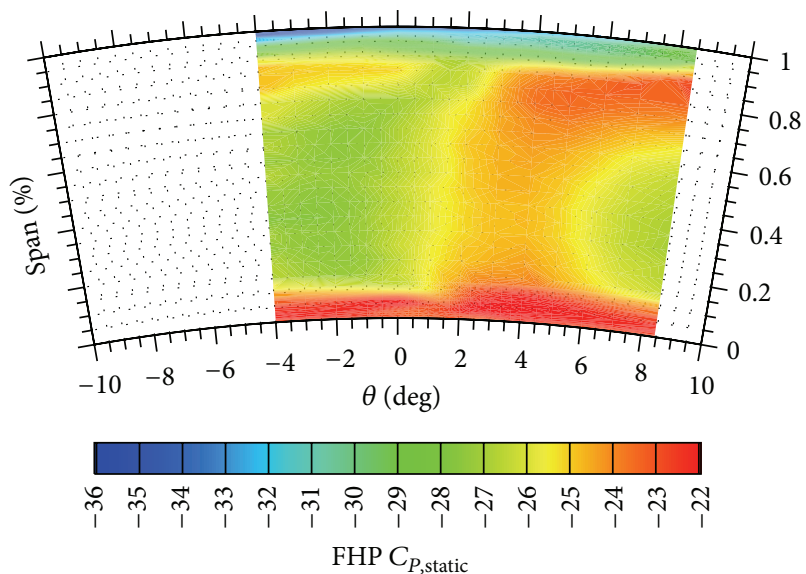

Figure 18: FHP coefficient of static pressure, intraspace.

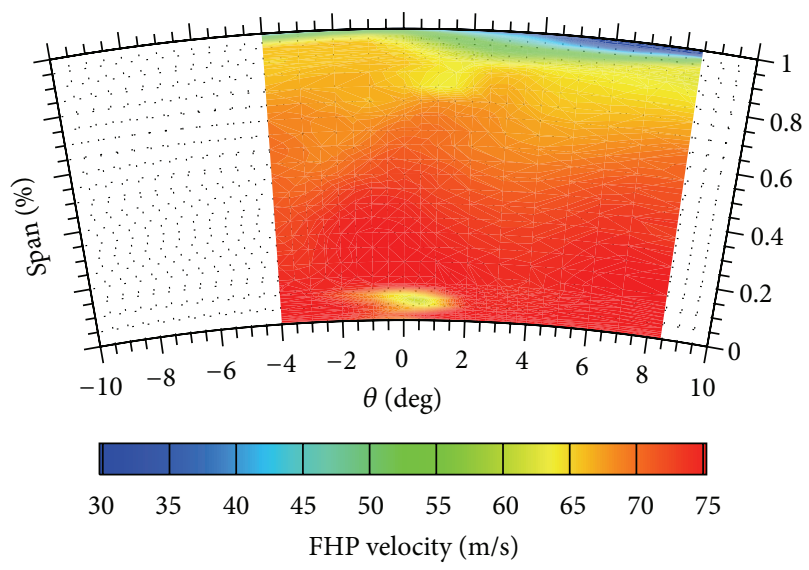

FIGURE 19: FHP velocity measurement, intraspace.

Since local velocity is known at each measurement location, mass averaging can be performed with the FHP results. This is achieved by taking the entire measurement area, partitioning it into slices of constant radius, and then finding the mass average of each slice of constant radius. Mass averaged total and static pressure are reported in Figure 20. The effect of the hub passage vortex is seen in the five to ten percent span region causing a higher static pressure and a lower total pressure. For much of the span the total pressure remains near zero, while static pressure is becoming greater as it moves toward the casing. Near the casing total and static pressure measurements converge due to loss from the casing boundary layer, passage vortex, and the increased uncertainty of the instrumentation slot.

Figure 21 shows the pitch angle measured by the FHP. In this case, a positive pitch angle means the flow is moving radially toward the center of the test rig, or from the casing to the hub. Here, the effects of the backward facing step can be seen as an increased alpha near the hub. The entire passage shows the flow is moving from the casing toward the hub as shown in Figure 20. Interference from the instrumentation slot results in flow moving radially toward the hub near $100 \%$ span.

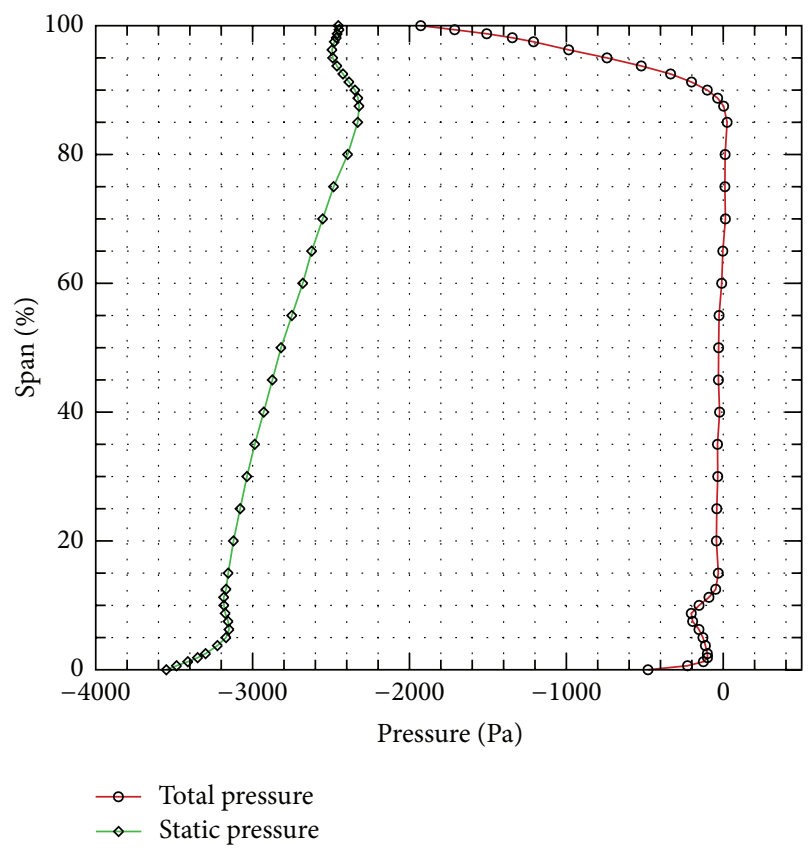

FIGURE 20: Mass averaged FHP measured total and static pressure, intraspace.

Mass averaged yaw angle is shown in Figure 22. The effect of the hub passage vortex is seen near $5 \%$ span as a quick increase and decrease in yaw angle. Near 100\% span, a yaw angle decrease is seen from the casing boundary layer and casing passage vortex. Increased uncertainty in this region is due to the instrumentation slot.

The mass averaged components of velocity are shown in Figure 23. Azimuthal velocity $\beta$ is the largest component of velocity. The fluid out of the NGV is highly swirled. A reduction in this velocity from the hub passage vortex can be seen from five to ten percent. Velocity near the casing boundary layer, casing passage vortex, and instrumentation slot locally reduces the azimuthal component of velocity. A nearly constant and very small magnitude radial velocity is shown over the entire span, signifying the flow is moving toward the hub from the casing. Axial velocity is the highest near the hub passage vortex, slowly changes over the whole span, and then quickly drops in the presence of the casing boundary layer and instrumentation slot. The velocity magnitude closely follows the azimuthal component. The effects of the hub passage vortex can be found from five to ten percent, and the casing effects are also shown as a reduction in velocity.

\section{Conclusions}

A subminiature Five-Hole Probe is calibrated using a newly designed automated pitch and yaw calibration system and an overview of various recent FHP implementations is given. The automated system reduces the amount of time for an 81point $(9 \times 9)$ carpet map calibration from three hours to 65 minutes. 


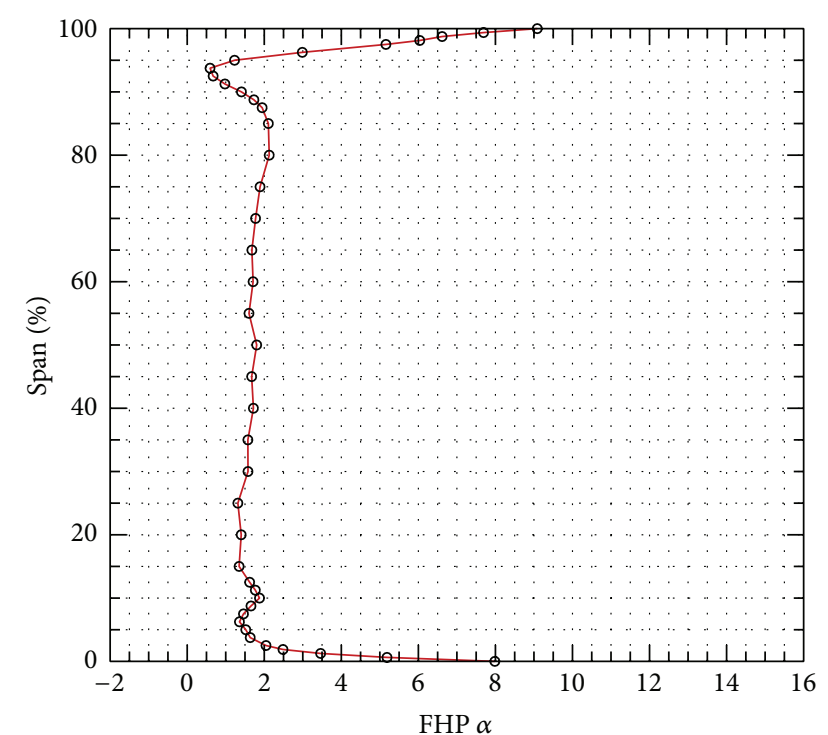

FIGURE 21: Mass averaged FHP measured pitch $(\alpha)$ angle, intraspace.

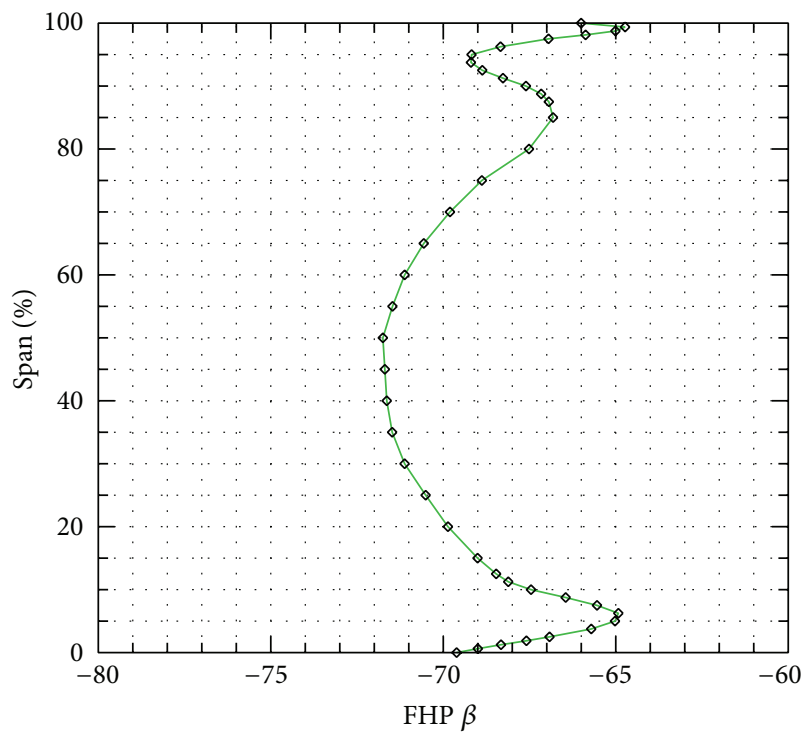

Figure 22: Mass averaged FHP measured yaw $(\beta)$ angle, intraspace.

Ensemble averaging of multiple runs measurably reduces the uncertainty of FHP calibrations in carpet maps. Uncertainty is further reduced by realigning the probe at a zero pitch, zero yaw position with the help of a laser level and plumb bob for each calibration run.

Using only one transducer during calibration reduces calibration uncertainty by eliminating the zero and calibration terms.

The fully automated pitch and yaw calibrator is designed to reduce uncertainty caused by streamwise displacement of the probe by keeping the tip of the probe close to the intersection of the pitch and yaw rotational axis. The pitch and yaw calibrator uses state-of-the-art mechanical components, stepper motors, indexers, and computer controls to provide

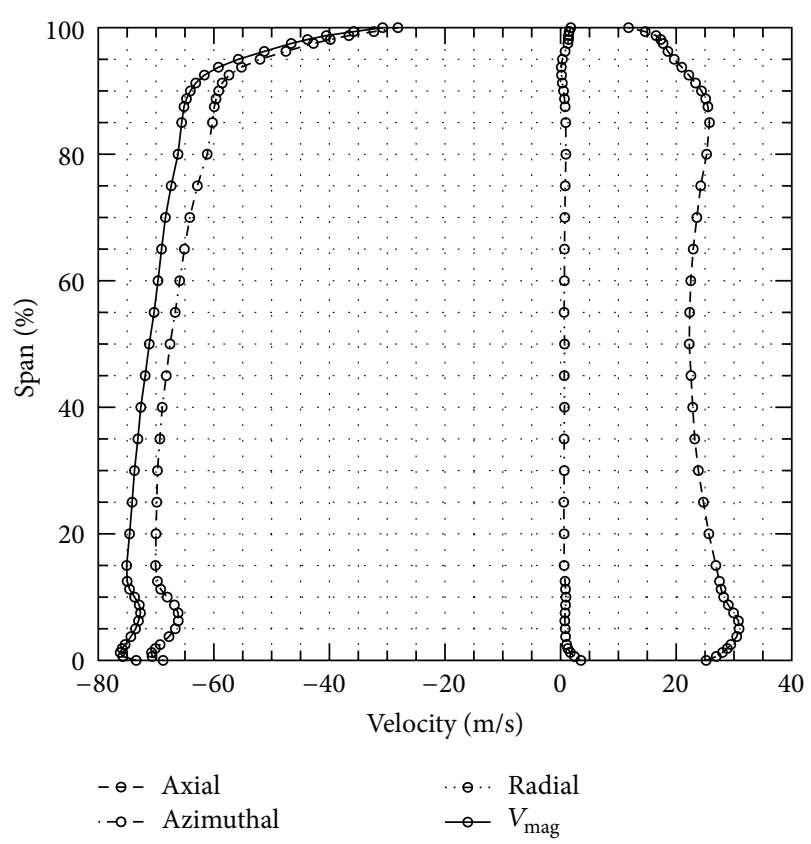

FIGURE 23: Mass averaged FHP measured velocity components, intraspace.

incremental angular changes in reduced time duration when compared to the conventional manual calibrator.

An uncertainty analysis is completed on the FHP calibration data. Within a $\pm 20^{\circ}$ range total pressure uncertainty is found to be less than $\pm 12 \mathrm{~Pa}$, static pressure uncertainty is less than $\pm 5 \mathrm{~Pa}$, and velocity uncertainty is less than $\pm 1 \mathrm{~m} / \mathrm{s}(4 \%)$.

A calibrated probe is placed into a large-scale axial turbine research rig. The research rig AFTRF has a recently modified probe traverser, state-of-the-art stepper motor drivers, pressure transducers, and programing approach. The new traverser replaces the old belt driven traverser with a mechanical system operated by a few linear translation stages. The new traversing system allows for effective adaptive gridding with much higher spatial resolution and position accuracy when compared to the belt driven system. The new system has increased the number of data points that can be collected in a two-hour turbine run period from 336 points to 868 points, an increase of approximately $160 \%$. Current system parameters are monitored in real-time using a graphical user interface allowing for ease of tracking and monitoring the results of the test.

The current adaptive grid definition is proved to the computer by the user in a manual manner. However, the present system also allows a fully automatic/adaptive grid definition starting from a coarse grid measurement. The present system has capabilities to detect large and small gradients of measured quantities in a relatively coarse grid. The computerized approach can define the new time optimized adaptive grid without human intervention. Experiments of this fully automated measurement grid generation system are underway.

The current approach provides a much needed mass averaged flow measurement system since it can provide all 
three components of the velocity vector over an area of interest with improved accuracy.

The calibrated probe currently maps the flow field behind the NGV using the new traversing system along one vane pitch and full span. Typical NGV passage exit flow structures such as vane wake, boundary layer, and passage vortex within the field are identified. The adaptive gridding method allows for measurements locations in these flow structures to be increased, while areas of smaller gradients are coarser. This leads to a reduction in the amount of time required to measure one entire vane passage.

The present paper presents significant improvements in FHP based aerodynamic measurements in four significant areas. The specific approach reduces the elapsed calibration time of a typical Five-Hole Probe. A second major improvement is in the spatial resolution of measurements in selected high gradient areas. The third important property of the present approach is in the improved accuracy of the measurements. Finally, the current approach reduces turbine facility run-time significantly.

\section{Conflict of Interests}

The authors declare that there is no conflict of interests regarding the publication of this paper.

\section{Acknowledgments}

The authors acknowledge the financial support provided for Jason Town by the Department of Aerospace Engineering at Penn State University. Cengiz Camci also acknowledges the support generously provided to him by TUBITAK, The Scientific and Technological Research Council of Turkey, during his sabbatical leave at Istanbul Technical University. Mr. Rick Auhl, Mark Catalano, and Kirk Hellen of Aerospace Engineering at Penn State provided significant technical expertise during the construction and execution of the experiments. The Five-Hole Probe construction at the subminiature scale would not have been possible without Mr. Harry Houtz's technical contributions.

\section{References}

[1] B. G. Wiedner, Passage Flow Structure and its Influence on Endwall Heat Transfer in a 90 Turning Duct, The Pennsylvania State University, University Park, Pa, USA, 1994.

[2] A. L. Treaster and A. M. Yocum, "The calibration and application of five-hole probes," ISA Transactions, vol. 18, no. 3, pp. 2334, 1979.

[3] C. Ostowari and W. H. Wentz, "Modified calibration techniques of a five-hole probe for high flow angles," Experiments in Fluids, vol. 3, no. 1, pp. 166-168, 1983.

[4] C. F. R. Nowack, "Improved calibration method for a fivehole spherical Pitot probe," Journal of Physics E: Scientific Instruments, vol. 3, no. 1, pp. 21-26, 1970.

[5] J. P. Weiz, An Algorithm for Using the Five-Hole Probe in the Non-Nulled Mode, The Applied Research Laboratory of the Pennsylvania State University, University Park, Pa, USA, 1980.
[6] B. A. Reichert and B. J. Wendt, A New Algorithm for Five-Hole Probe Calibration and Data Reduction and Its Application to a Rake-Type Probe, ASME-FED, 1993.

[7] R. G. Dominy and H. P. Hodson, "Investigation of factors influencing the calibration of five-hole probes for three-dimensional flow measurements," Journal of Turbomachinery, vol. 115, no. 3, pp. 513-519, 1993.

[8] G. L. Morrison, M. T. Schobeiri, and K. R. Pappu, "Fivehole pressure probe analysis technique," Flow Measurement and Instrumentation, vol. 9, no. 3, pp. 153-158, 1998.

[9] S. W. Lee and T. J. Yoon, "An investigation of wall-proximity effect using a typical large-scale five-hole probe," Korean Society of Mechanical Engineers International Journal, vol. 3, no. 13, pp. 273-285, 1999.

[10] M. C. Brophy, A. L. Treaster, D. R. Stinebring, and J. P. Weiz, "Optimization of a five-hole probe wake measuring system," Technical Memorandum 87-156, Applied Research Laboratory, The Pennsylvania State University, University Park, Pa, USA, 1980.

[11] N. Sitaram, B. Lakshminarayana, and A. Ravindranath, "Convential probes for the relative flow measurement in a rotor blade passage," in Proceedings of the Joint Fluids Engineering Gas Turbine Conference and Products Show, New Orleans, La, USA, March 1980.

[12] J. Town and C. Camci, "Sub-miniature five-hole probe calibration using a time efficient pitch and yaw mechanism and accuracy improvments," in Proceedings of the ASME International Gas Turbine Conference, ASME GT-46391, Vancouver, Canada, 2011.

[13] A. J. Pisasale and N. A. Ahmed, "Theoretical calibration for highly three-dimensional low-speed flows of a five-hole probe," Measurement Science and Technology, vol. 13, no. 7, pp. 11001107, 2002.

[14] A. J. Pisasale and N. A. Ahmed, "A novel method for extending the calibration range of five-hole probe for highly threedimensional flows," Flow Measurement and Instrumentation, vol. 13, no. 1-2, pp. 23-30, 2002.

[15] A. J. Pisasale and N. A. Ahmed, "Development of a functional relationship between port pressures and flow properties for the calibration and application of multihole probes to highly threedimensional flows," Experiments in Fluids, vol. 36, no. 3, pp. 422-436, 2004.

[16] S. J. Lien and N. A. Ahmed, "An examination of suitability of multi-hole pressure probe technique for skin friction measurement in turbulent flow," Flow Measurement and Instrumentation, vol. 22, no. 3, pp. 215-224, 2011.

[17] K. Kuisoon, B. G. Wiedner, and C. Camci, "Turbulent flow and endwall heat transfer analysis in a 90。 turning duct and comparisons with measured data-part II: influence of secondary flow, vorticity, turbulent kinetic energy, and thermal boundary conditions on endwall heat transfer," International Journal of Rotating Machinery, vol. 8, no. 2, pp. 125-140, 2002.

[18] K. Kuisoon, B. G. Wiedner, and C. Camci, "Turbulent flow and endwall heat transfer analysis in a $90^{\circ}$ turning duct and comparisons with measured data. Part I. Influence of Reynolds number and streamline curvature on viscous flow development," International Journal of Rotating Machinery, vol. 8, no. 2, pp. 109-123, 2002.

[19] C. Camci and D. H. Rizzo, "Secondary flow and forced convection heat transfer near endwall boundary layer fences in a $90^{\circ}$ turning duct," International Journal of Heat and Mass Transfer, vol. 45, no. 4, pp. 831-843, 2001. 
[20] B. Lakshminarayana, C. Camci, I. Halliwell, and M. Zaccaria, "Design and development of a turbine research facility to study rotor-stator interaction effects," International Journal of Turbo \& Jet Engines, vol. 13, no. 3, pp. 155-172, 1996.

[21] J. R. Taylor, An Introduction to Error Analysis the Study of Uncertainties and Physical Measurements, University Science Books, Sausalito, Calif, USA, 1997. 

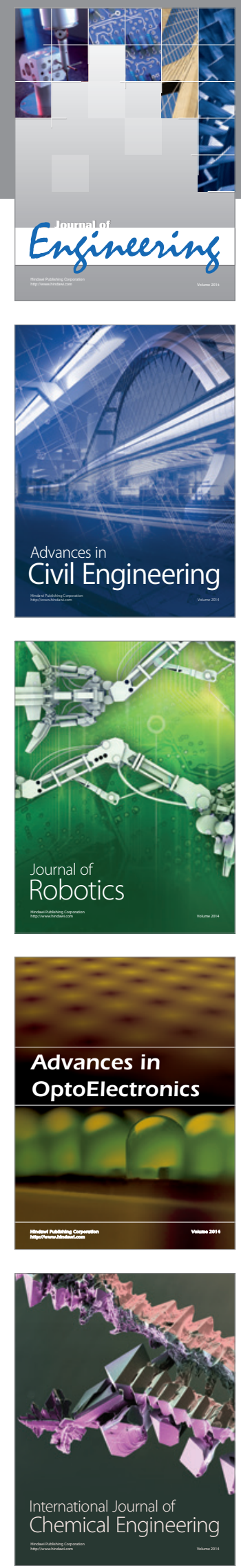

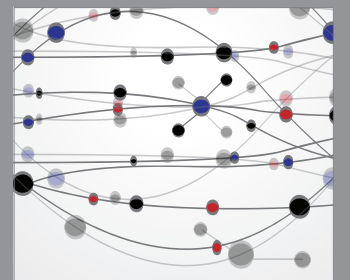

The Scientific World Journal
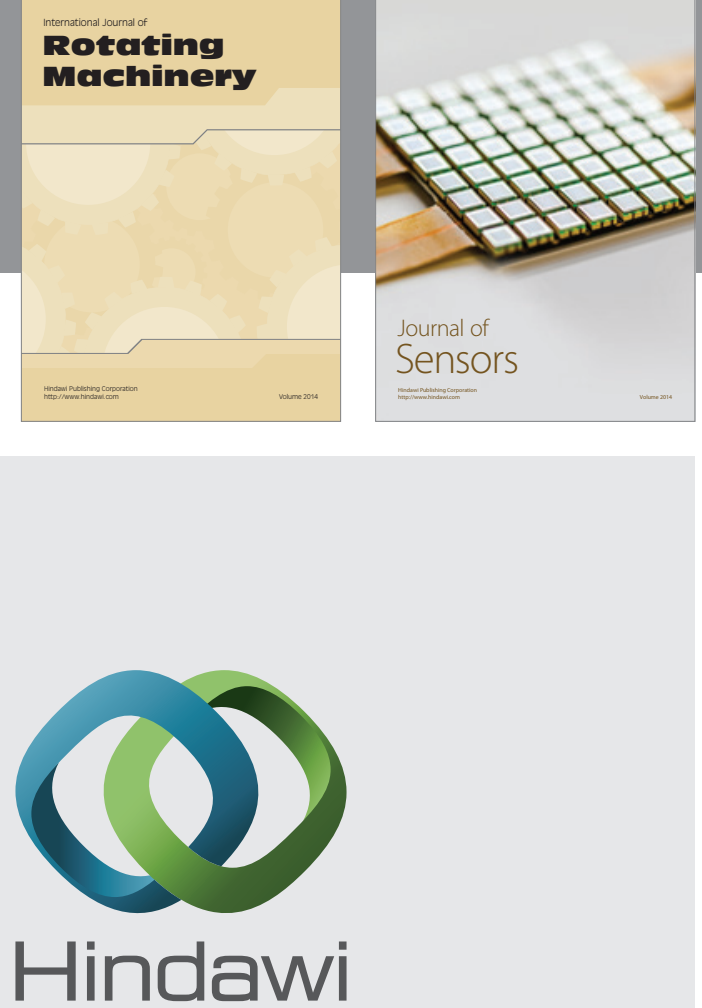

Submit your manuscripts at http://www.hindawi.com
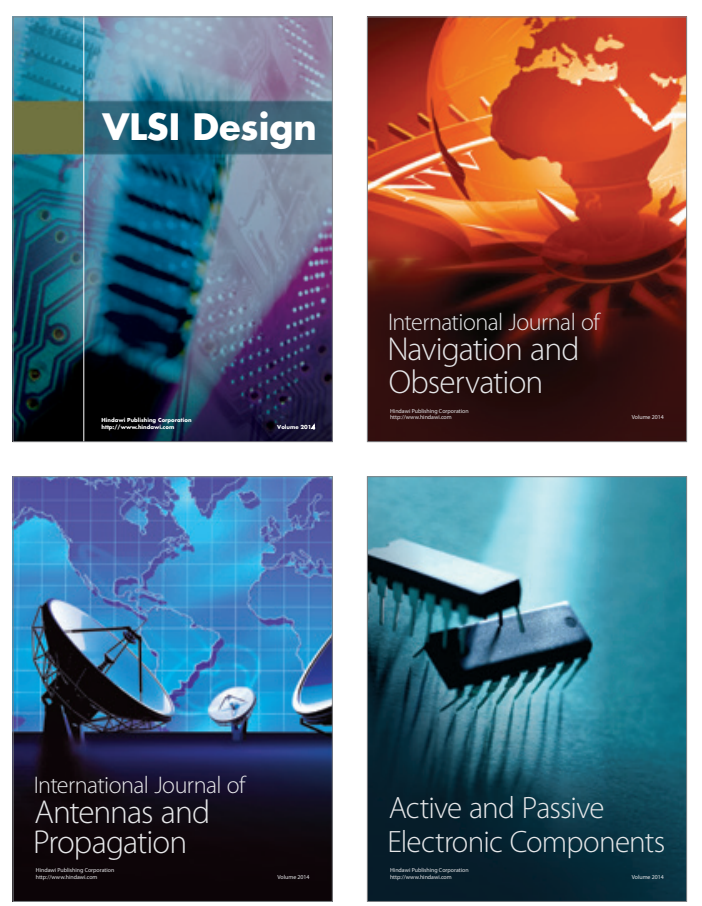
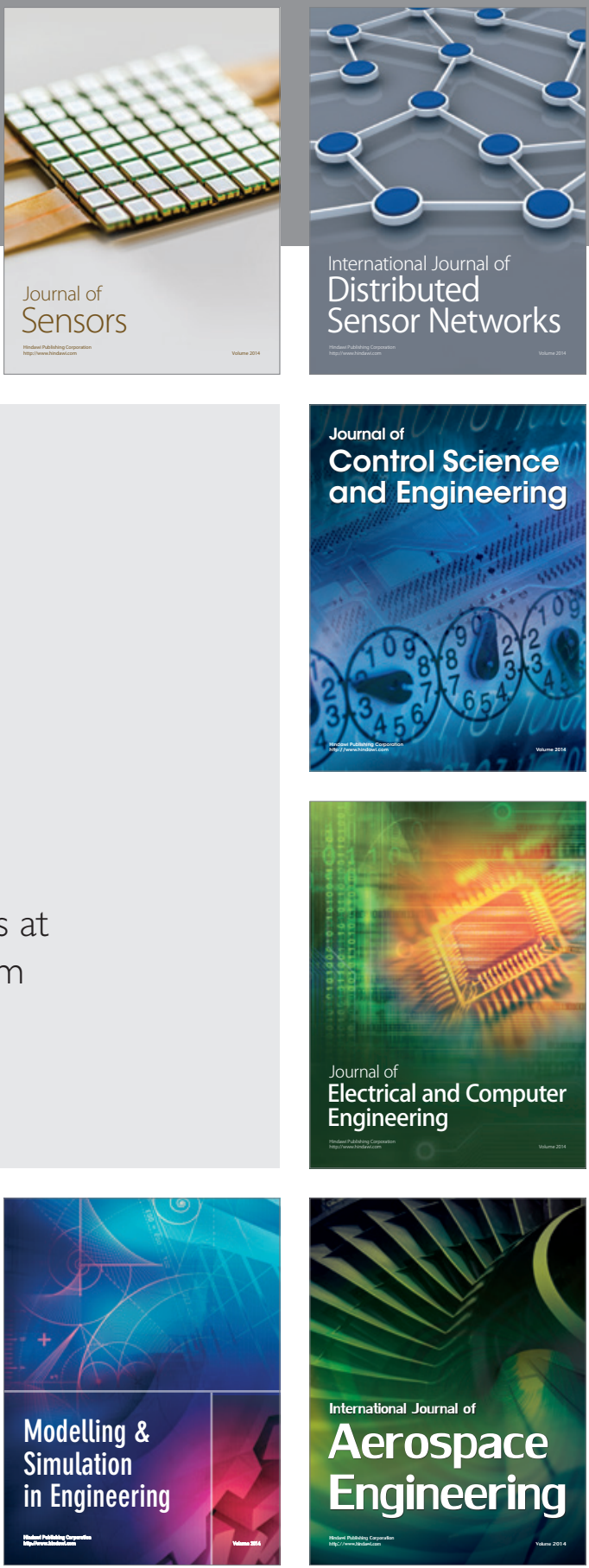

Journal of

Control Science

and Engineering
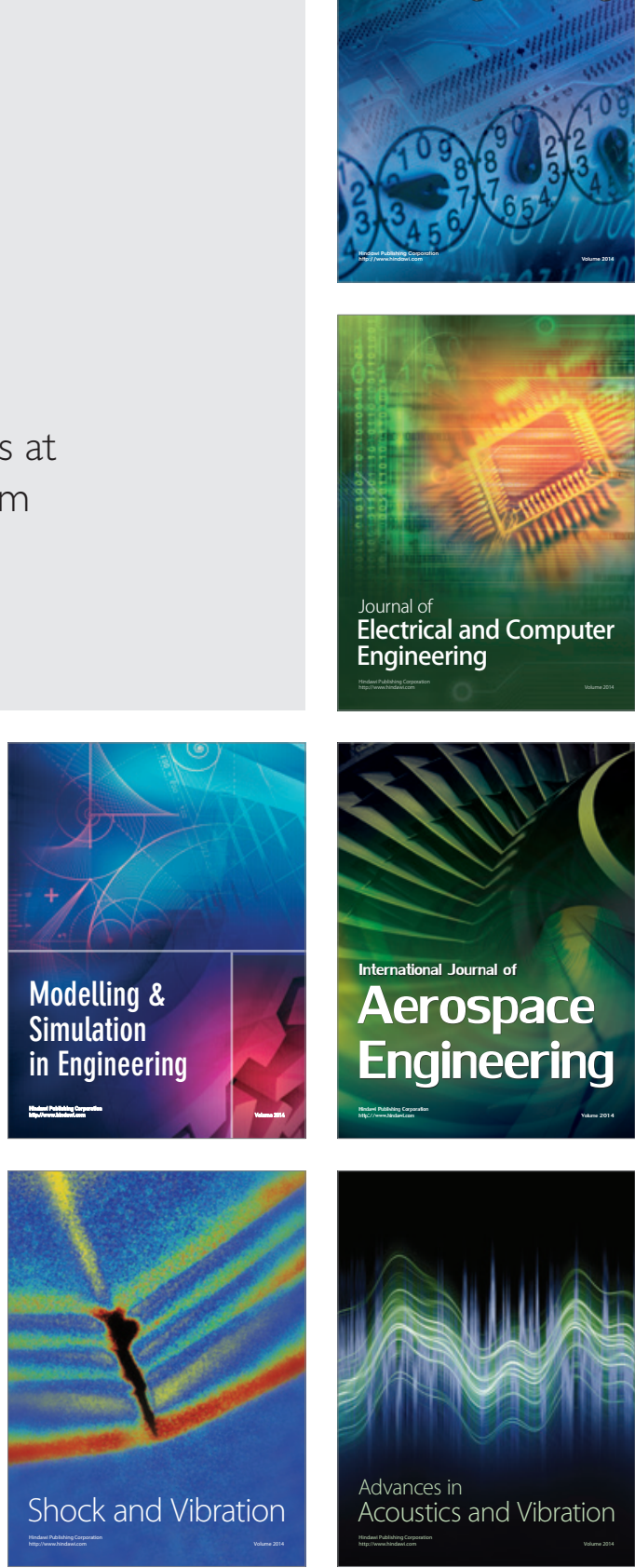